\title{
A consecutive-interpolation finite element method for heat transfer analysis
}

- Nguyen Ngoc Minh ${ }^{1}$

- Nguyen Thanh Nha ${ }^{1}$

- Bui Quoc Tinh ${ }^{2}$

- Truong Tich Thien ${ }^{1}$

${ }^{1}$ Ho Chi Minh city University of Technology, VNU-HCM

2 Dept. of Mechanical and Environmental Informatics, Tokyo Institute of Technology, 2-12-1-W8-22, Ookayama, Meguro-ku, Tokyo, 152-8552, Japan

(Manuscript Received on August 01 ${ }^{\text {st }}$, 2015, Manuscript Revised August 27 ${ }^{\text {th }}$, 2015)

\section{ABSTRACT:}

A consecutive-interpolation 4-node quadrilateral finite element (CQ4) is further extended to solve twodimensional heat transfer problems, taking the average nodal gradients as interpolation condition, resulting in highorder continuity solution without smoothing operation and without increasing the number of degrees of freedom. The implementation is straightforward and can be easily integrated into any existing FEM code. Several numerical examples are investigated to verify the accuracy and efficiency of the proposed formulation in two-dimensional heat transfer analysis.

Key words: heat transfer, CFEM, conduction, convection, nodal gradients.

\section{INTRODUCTION}

Heat transfer analysis is of great importance to both engineering and daily life, as one may encounter the problem of heat transfer almost in every activities, such as heating, cooling, air convection etc. Since analytical solutions are only available for some restricted problems, in most cases one has to rely on numerical methods to perform analysis.

The standard finite element method (FEM) has been successfully used for heat transfer problems. However, despite its simplicity, the FEM still has many inherent shortcomings. The FEM shape function is $\mathrm{C}^{0}$-continuous, thus the nodal gradient fields, i.e., the temperature gradient in case of heat transfer, is discontinuous across element boundaries. More critically, FEM suffers loss of accuracy when the mesh is heavily distorted [1].

Various alternative methods have been proposed to overcome the difficulties raised by FEM. A class of meshfree methods like the Element Free Galerkin method (EFG) [2], the meshless local Petrov-Galerkin method [3] and the Radial Point Interpolation method [4] are used for investigating heat transfer problems. The meshfree methods offer flexibility due to the fact that only nodes are required, which would be great advantage in case the re-meshing is necessary. However, the disadvantages of meshfree methods include the complexity in the calculation of meshfree shape functions, and the lack of Kronecker delta property leading to 
difficulties in the imposition of essential boundary conditions. Another trend is the application of smoothing technique into existing numerical methods, such as the smoothed finite element method (SFEM) [1] and the smoothed radial point interpolation method [5]. In this class of methods, the smoothing operator is applied during the calculation of gradient fields, i.e., the strain in mechanical problems and temperature gradient in heat transfer problems, leading to the modification of the "stiffness" matrices. The nodal gradient field is thus continuous.

Recently, a new consecutive-interpolation method (CFEM) has been proposed [6, 7], in which the conventional approximation used in FEM is modified such that the averaged nodal gradients are taken into account. The nodal gradient field is thus continuous, but unlike the SFEM, no smoothing technique is required. In post-processing, stress-recovery, which is quite often used in terms of the FEM, is not necessary. One interesting point is that there are no additional degrees of freedom to the system. The problem size remains the same as that in the FEM. Inspired by the advantages and potential of CFEM reported for the linear elastic problems [6, 7], this particular research further extends the method by investigating its capabilities in heat transfer analysis.

The outline of the paper is as follows. A brief on CFEM formulation for heat transfer problems is reported in Section 2. Section 3 presents the numerical examples, in which the capabilities of CFEM in heat transfer analysis are numerically illustrated. Conclusions and remarks are given in Section 4.

\section{CFEM FOR HEAT TRANSFER PROBLEMS}

\subsection{Brief on CFEM}

Let us consider a $2 \mathrm{D}$ body in the domain $\Omega$ bounded by $\Gamma=\Gamma_{\mathrm{u}}+\Gamma_{\mathrm{t}}$ và $\Gamma_{\mathrm{u}} \cap \Gamma_{\mathrm{t}}=\{\}$. Using the Finite Element Method (FEM), the domain $\Omega$ is discretized into non-overlapping sub-domains $\Omega_{\mathrm{e}}$ called elements. The elements are interconnected at points called nodes. Any function $u(\mathbf{x})$ with $\mathbf{x} \in \Omega$ can be approximated by

$$
u(\mathbf{x}) \approx \tilde{u}(\mathbf{x})=\sum_{i=1}^{n} N_{i}(\mathbf{x}) \hat{u}_{i}=\mathbf{N} \hat{\mathbf{u}}
$$

where $\hat{\mathbf{u}}$ is the vector of nodal values, $n$ is the number of nodes, $\mathbf{N}$ is the vector of shape functions and $N_{i}$ is the shape function associated with node $i$. The standard approximation scheme within a finite element, $e$, is then given by

$$
\tilde{u}^{e}(\mathbf{x})=\sum_{i=1}^{n e} N_{i}(\mathbf{x}) \hat{u}_{i}=\mathbf{N} \hat{\mathbf{u}}^{e},
$$

where $n e$ is the number of nodes of the element. By assigning the approximated value at node $i$ as $u^{[i]}=u\left(\mathbf{x}_{i}\right)$, and the vector of shape functions evaluated at node $i$ as $\mathbf{N}^{[i]}=\mathbf{N}\left(\mathbf{x}_{i}\right)$, the average nodal derivatives $u_{x}^{[i]}$ (similar to $\left.u_{, y}^{[i]}\right)$ can then be determined by $[6,7]$

$\bar{u}_{, x}^{[i]}=\overline{\mathbf{N}}_{, x}^{[i]} \hat{\mathbf{u}}$,

where $\overline{\mathbf{N}}_{x}^{[i]}$ are the averaged derivative of $\mathbf{N}^{[i]}$, which are calculated by

$$
\overline{\mathbf{N}}_{, x}^{[i]}=\sum_{e \in S_{i}}\left(w_{e} \cdot \mathbf{N}_{, x}^{[i][e]}\right),
$$

with $\mathbf{N}_{, x}^{[i][e]}$ being the derivative of $\mathbf{N}^{[i]}$ computed in element $e$. In Eq. (4), $S_{i}$ is the the set of elements containing all the elements connected to node $i$, while $\mathrm{w}_{\mathrm{e}}$ is a weight function dependent on the element type and is defined as in [7]

$$
w_{e}=\frac{\Delta_{e}}{\sum_{\bar{e} \in S_{i}} \Delta_{\bar{e}}}
$$

with and $\Delta_{e}$ being the area of element $e \in S_{i}$

One well-known shortcoming of the standard FEM is the discontinuity of strains and stresses due to the discontinuity of the nodal gradients. In the CIP approach, both the nodal 
values $u^{[i]}$ and the averaged nodal derivatives $\bar{u}_{, x}^{[i]}$ (and $\bar{u}_{, y}^{[i]}$ ) are taken into the interpolations, which can substantially overcome such drawback of the discontinuity in the stress and strain fields. As a consequence, the approximation in Eq. (1) can be rewritten by means of the CIP scheme as follows

$$
\begin{aligned}
\tilde{u}(\mathbf{x}) & =\sum_{i=1}^{n}\left(\phi_{i} u^{[i]}+\phi_{i x} \bar{u}_{, x}^{[i]}+\phi_{i y} \bar{u}_{, y}^{[i]}\right) \\
& =\sum_{i=1}^{n}\left(\phi_{i} \mathbf{N}^{[i]}+\phi_{i x} \overline{\mathbf{N}}_{, x}^{[i]}+\phi_{i y} \overline{\mathbf{N}}_{, y}^{[i]}\right) \hat{\mathbf{u}} \\
& =\tilde{\mathbf{N}}(\mathbf{x}) \hat{\mathbf{u}}
\end{aligned}
$$

where

$\tilde{\mathbf{N}}(\mathbf{x})=\sum_{i=1}^{n}\left(\phi_{i} \mathbf{N}^{[i]}+\phi_{i x} \overline{\mathbf{N}}_{, x}^{[i]}+\phi_{i y} \overline{\mathbf{N}}_{, y}^{[i]}\right)$ is the CIP shape functions, in which $\phi_{i}, \phi_{i x}$ and $\phi_{i y}$ are the field functions dependent on the element type. Applying the CIP approach to the constant strain triangular element (T3), one obtained the CT3 element [6] and the CQ4 element [7] is obtained from the standard bilinear quadrilateral element (Q4).

Fig. 1 illustrates the application of CIP

approach into Q4 element described particularly in an irregular finite element mesh, in which the sets $S_{i}, S_{\mathrm{j}}, S_{k}, S_{m}$ contain all the neighboring elements that share the node $i, j, k, m$, respectively. It indicates that the supporting nodes for the point of interest $\mathbf{x}$ include all the nodes in the element sets $S_{i}, S_{j}, S_{k}, S_{m}$. Thus, as shown in Fig. 1, the support domain in CFEM is in any cases larger than that of the standard FEM, since it includes not only the nodes of the element in interest but also the nodes of the adjacent elements. Using Eq. (2), (3) and (6), the interpolation scheme for a CQ4 element for any point $\mathbf{x}$ can then be expressed by

$$
\tilde{u}^{e}(\mathbf{x})=\sum_{l=i, j, k, m}\left(\phi_{l} u^{[l]}+\phi_{l x} \bar{u}_{, x}^{[l]}+\phi_{l y} \bar{u}_{, y}^{[l]}\right) .
$$

In Eq. (7), the field functions $\phi_{i}, \phi_{i x}$ are calculated as follows [7]

$$
\begin{aligned}
\phi_{i}= & L_{i}+L_{i}^{2}\left(L_{j}+L_{k}+L_{m}\right)-L_{i}\left(L_{j}^{2}+L_{k}^{2}+L_{m}^{2}\right) \\
\phi_{i x}= & -\left(x_{i}-x_{j}\right)\left(L_{i}^{2} L_{j}+p L_{i} L_{j} L_{k}+p L_{i} L_{j} L_{m}\right) \\
& -\left(x_{i}-x_{k}\right)\left(L_{i}^{2} L_{k}+p L_{i} L_{k} L_{m}+p L_{i} L_{k} L_{j}\right), \\
& -\left(x_{i}-x_{m}\right)\left(L_{i}^{2} L_{m}+p L_{i} L_{m} L_{j}+p L_{i} L_{m} L_{k}\right)
\end{aligned}
$$

in which $L_{\mathrm{i}}, L_{\mathrm{j}}, L_{\mathrm{k}}$ and $L_{m}$ are the shape functions of the element $\mathrm{Q} 4$ and $p=0.5$. The function $\phi_{i y}$ is obtained simply by replacing the $x$-coordinates in equation (9) by the corresponding $\mathrm{y}$-coordinates. The functions $\phi_{j}, \phi_{j x}$ and $\phi_{j y} ; \quad \phi_{k}, \phi_{k x}$ and $\phi_{k y} ; \phi_{m}, \phi_{m x}$ and $\phi_{m y}$ can be computed in the similar manner by a cyclic permutation of indices $i, j, k, m$.

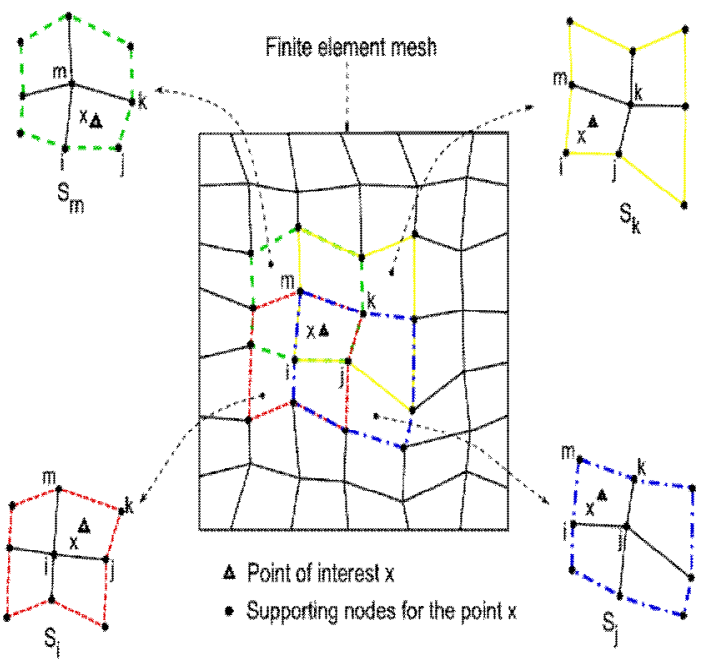

Figure 1. Schematic sketch of CQ4 element

\subsection{Desirable properties of the shape functions}

Figs. $2 \mathrm{a}$ and $2 \mathrm{~b}$ show a comparison of the 1D shape functions and the first-order derivatives between FEM and CFEM. The CQ4 shape function and the first-order derivative are depicted in Fig. 3. It is observed that the CFEM shape functions and their first-order derivatives 
are smoother than the FEM counterparts. In particular, the CFEM shape functions are $C^{l}$ continuity on nodes, allowing the strain and stress fields to be continuous across the nodes. Unlike some other higher-order continuity methods, such as the Element-free Galerkin method or Isogeometric Analysis, the CFEM possesses the Kronecker-delta property, enabling direct imposition of boundary conditions. It is also worth mentioning that the unknowns contain only the nodal displacement, as shown in Eq. (6). Thus, no additional degrees of freedom are required.

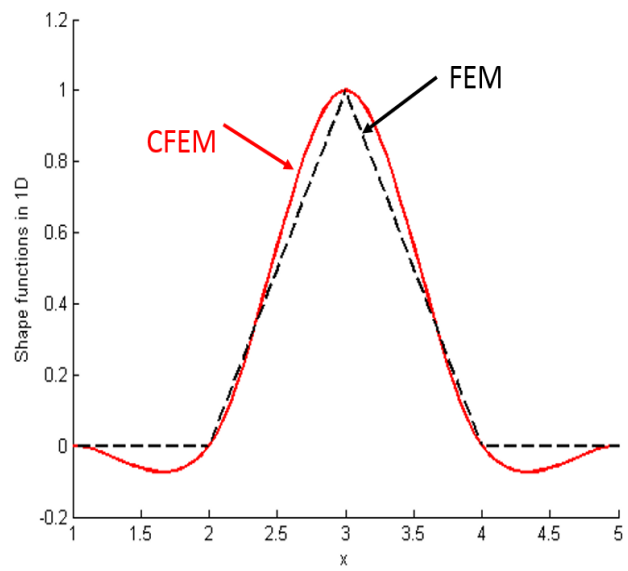

(a)

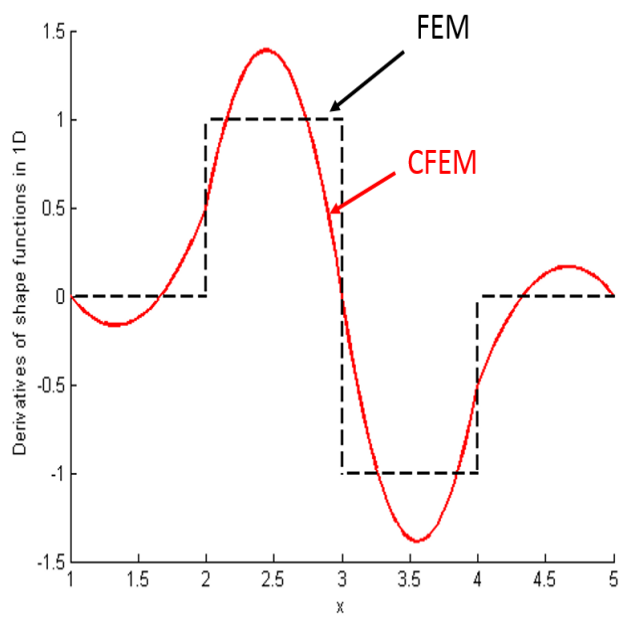

(b)

Figure 2. Comparison of (a) one-dimensional shape functions and (b) their first-order derivatives between CFEM and FEM

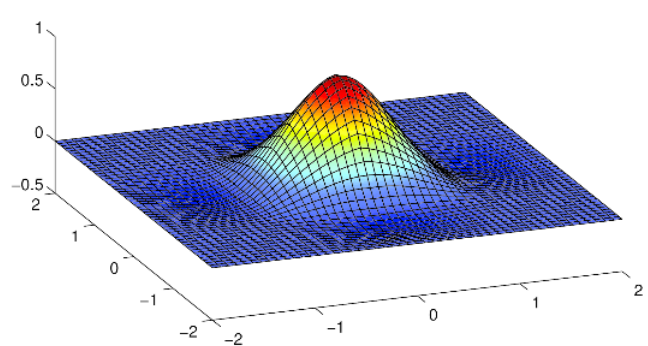

(a)

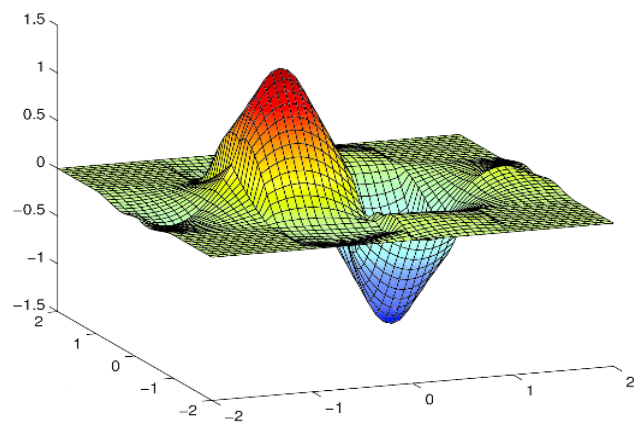

(b)

Figure 3. Visualization of (a) Shape function and (b) first-order derivative of CQ4 element

\subsection{Governing equations of heat transfer problems}

The governing equation of a heat transfer problem in a domain $\Omega$ is given by

$$
\nabla \cdot(k \nabla T)+Q=\rho c \dot{T},
$$

with the following boundary conditions

$T=\bar{T}$ on $\Gamma_{1}$ : essential boundary

$-k \nabla T=\bar{q}$ on $\Gamma_{2}$ : surfae heat flux boundary

$-k \nabla T=0$ on $\Gamma_{3}$ : adiabatic boundary (13)

$-k \nabla T=h\left(T_{a}-T\right)$ on $\Gamma_{4}:$ convective boundary

$$
-k \nabla T=\varepsilon \sigma\left(T_{a}^{4}-T^{4}\right) \text { on } \Gamma_{4}: \text { radiation }
$$
boundary

In Eqs. (10) to (15), $\mathbf{k}=\operatorname{diag}\left(k_{\mathrm{xx}}, k_{\mathrm{yy}}, k_{\mathrm{zz}}\right)$ is the tensor of thermal conductivities, $\mathrm{T}$ the temperature field, $\mathrm{Q}$ the body heat flux, $\rho$ the 
density and $c$ the specific heat capacity, $\mathrm{h}$ the convective coefficient, $T_{\mathrm{a}}$ the ambient temperature, $\sigma$ the Stefan-Boltzmann constant for radiation and $\varepsilon$ the emissivity which is taken as 1 in this paper. In case of steady-state analysis, the time derivative term vanishes. In general, the heat transfer problem is non-linear. If we consider constant thermal conductivities, constant convective coefficient and do not take radiation into account, the problem becomes linear. For the sake of simplicity, but without loss of generality, we consider linear steady-state heat transfer problems in this paper.

\section{NUMERICAL EXAMPLES}

\subsection{Heat conduction in 2D}

In this example, a two dimensional heat conduction problem is considered as depicted in Fig. 4. The surface heat flux $q=3000 \mathrm{~W} / \mathrm{m} 2$ is applied on the Neumann boundary $\Gamma 1$. On essential boundary $\Gamma 2$, the temperature is prescribed as $\mathrm{T}=303 \mathrm{~K}$. The thermal conductivity is given by $\mathrm{k}=20 \mathrm{~W} / \mathrm{mK}$. There is no analytical solution available for this problem, a FEM solution using a fine mesh, e.g.10000 Q4 elements, is taken for comparison purpose. The temperature distribution is given as in Fig. 5. Fig. 6 presents the equivalent thermal energy evaluated by CFEM for various discretization, for instance, $5 \times 5,10 \times 10,20 \times 20$ and $40 \times 40$ elements, showing the convergence tendency with respect to the number of degrees of freedom. It is observed in the results that even with a coarsest mesh of $5 \times 5$ elements, the numerical error is still very small, $0.09 \%$ and the result converges quickly to an upper bound of the reference one. Fig. 7 depicts a comparison of temperature gradient obtained by CFEM and FEM, respectively, highlighting the smoothness of CFEM solution, while a discontinuity across element is found for the FEM.

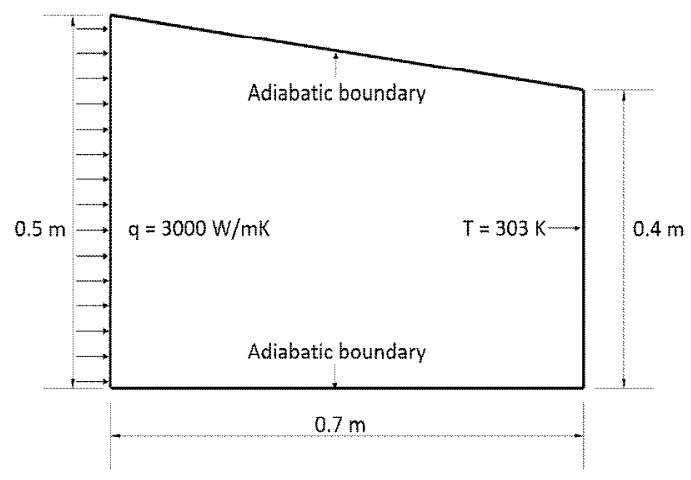

Figure 4. 2D heat conduction problem: model

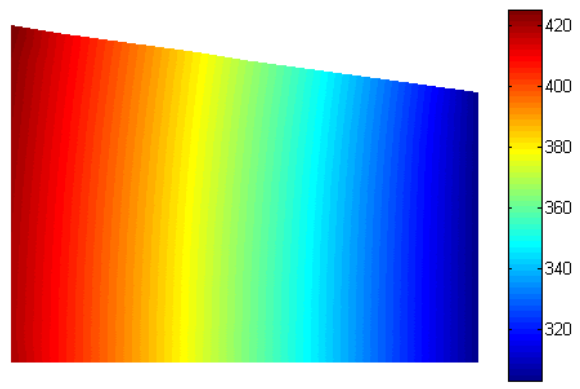

Figure 5. 2D heat conduction problem: temperature distribution

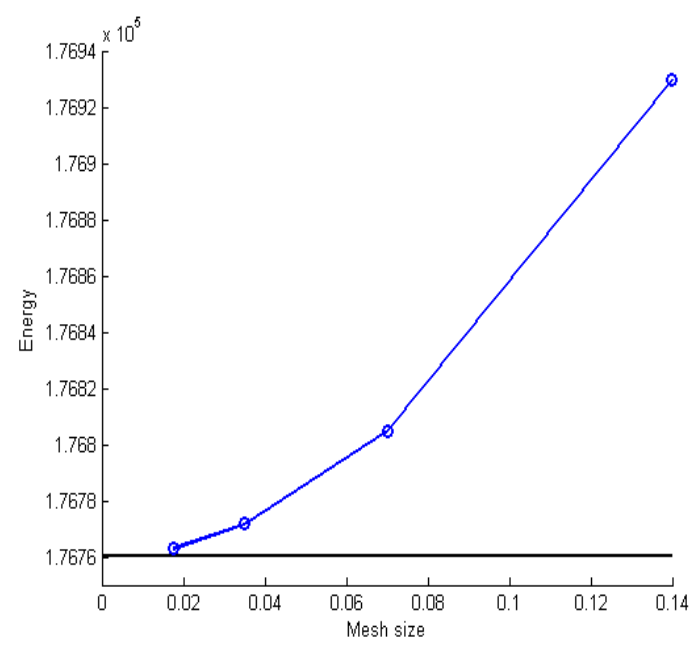

Figure 6. 2D heat conduction: convergence of the equivalent thermal energy with respect to mesh size 


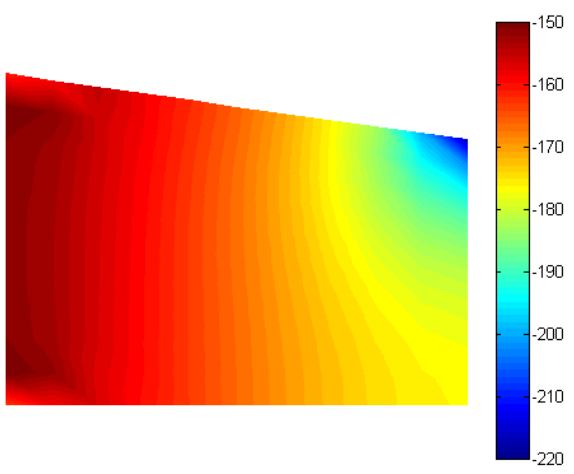

(a)

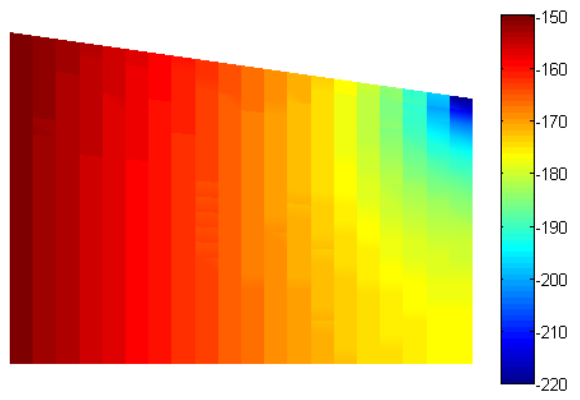

(b)

Figure 7. 2D heat conduction: Temperature gradient $\partial T / \partial x$ obtained by (a) CFEM and (b) FEM

\subsection{Heat convection in 2D}

This example deals with a more complicated problem of 2D heat transfer analysis where the model is sketched in Fig. 8a. The inward surface heat flux is defined as $\mathrm{q}=20000 \mathrm{~W} / \mathrm{m}^{2}$. The conductivity is $\mathrm{k}=100 \mathrm{~W} / \mathrm{mK}$. Convection is applied on the left hand side boundary with a coefficient $\mathrm{h}=100 \mathrm{~W} / \mathrm{m}^{2}$ and the ambient temperature is $\mathrm{T}_{\mathrm{a}}=300 \mathrm{~K}$. On the essential boundary, the temperature is prescribed as $\mathrm{T}=$ $300 \mathrm{~K}$. Again, no analytical solution is available for this particular example and we hence adopt a FEM solution using a fine mesh of 5936 Q4 elements as reference results.

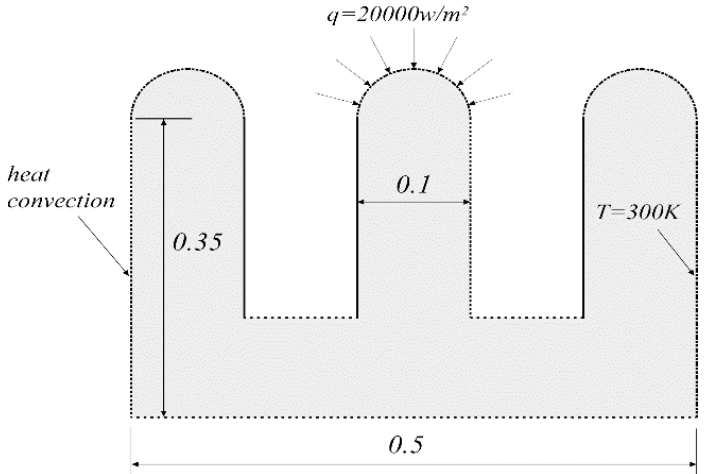

(a)

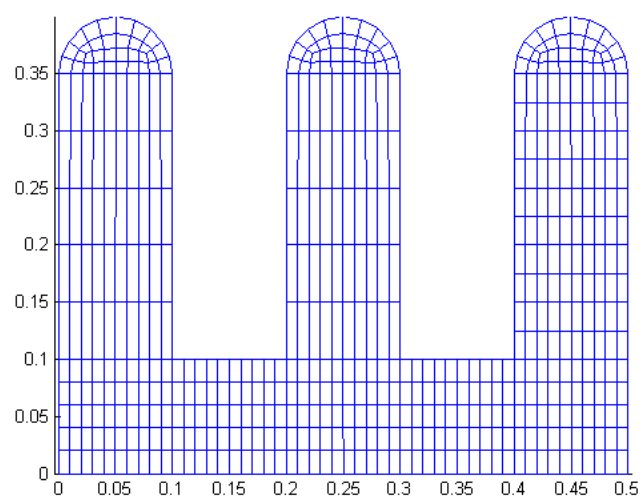

(b)

Figure 8. 2D heat convection: (a) Geometry and (b) Finite element mesh

The temperature distribution is illustrated in Fig. 9. A comparison of the maximum temperature (found at the Neumann boundary) obtained by CFEM and FEM, respectively, for the same mesh, are presented in Table 1. It is interesting to see that, given the same mesh, the CFEM solution is closer to the reference solution, showing the higher accuracy of CFEM as compared with the one derived from the FEM. The higher accuracy of CFEM is clear as it is due to the fact that the degree of shape functions of the CFEM is higher than that of the FEM. The nodal temperature gradient is plotted in Fig. 10, where one can observe that the CFEM solution is really smoother, which is again expected as observed in Example 3.1. 


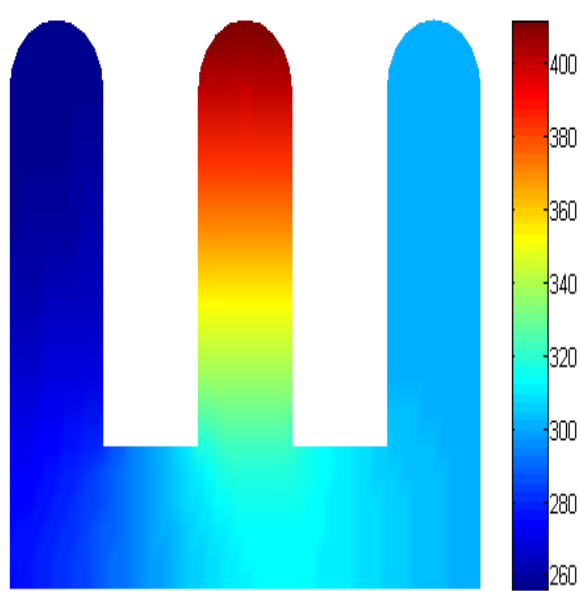

Figure 9. 2D heat convection: temperature distribution

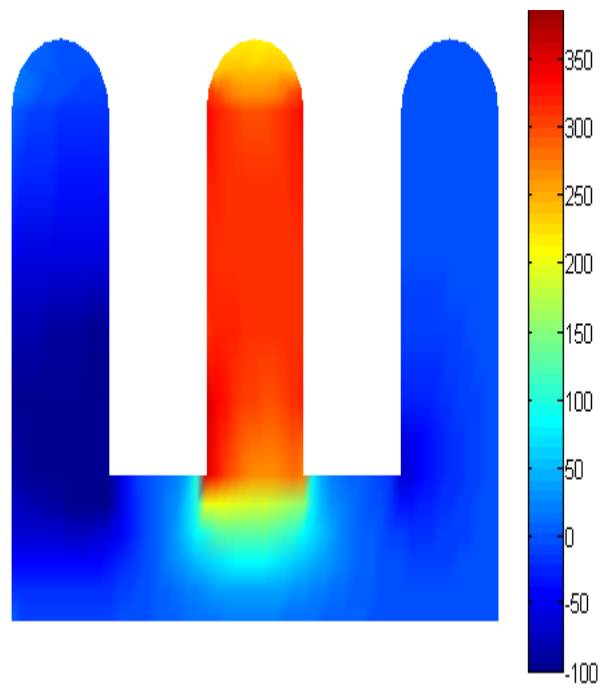

(a)

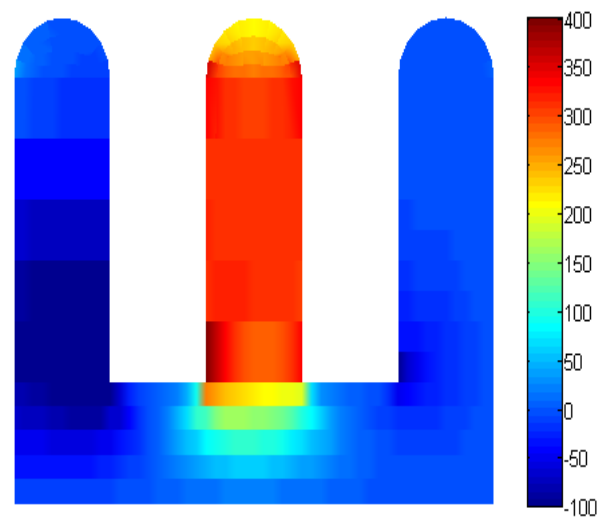

(b)

Figure 10. 2D heat convection: Temperature gradient $\partial T / \partial y$ obtained by (a) CFEM and (b) FEM

\section{CONCLUSIONS}

We have successfully extended the consecutive-interpolation finite element method (CFEM) to solve the heat transfer problem in 2D. The CFEM provides some advantages as follows:

Smooth temperature gradient without using any smoothing technique, while retaining increasing the number of degrees of freedom.

Higher accuracy due to the higher-order shape function.

Straightforward and easy to be implemented into any existing FEM code.

From this particular study, the CFEM is demonstrated as an effective numerical tool to be considered as an alternative to the standard FEM in modeling heat transfer problems.

Table 1. 2D Convection: Comparison of the maximum temperature. The relative errors with the reference results are displayed in brackets

\begin{tabular}{|c|c|c|c|c|}
\hline $\begin{array}{c}\text { FEM (219 Q4 } \\
\text { elements) }\end{array}$ & $\begin{array}{c}\text { CFEM (219 CQ4 } \\
\text { elements) }\end{array}$ & $\begin{array}{c}\text { FEM (546 Q4 } \\
\text { elements) }\end{array}$ & $\begin{array}{c}\text { CFEM (546 Q4 } \\
\text { elements) }\end{array}$ & $\begin{array}{c}\text { Reference (5936 Q4 } \\
\text { elements) }\end{array}$ \\
\hline $410.742 \mathrm{~K}$ & $410.844 \mathrm{~K}$ & $411.174 \mathrm{~K}$ & $411.215 \mathrm{~K}$ & $412.235 \mathrm{~K}$ \\
$(-0.36 \%)$ & $(-0.34 \%)$ & $(-0.26 \%)$ & $(-0.25 \%)$ & \\
\hline
\end{tabular}




\section{Phương pháp phần tử hữu hạn nội suy liên tiếp trong phân tích truyền nhiệt}

- Nguyễn Ngọc Minh ${ }^{1}$

- Nguyễn Thanh Nhã ${ }^{1}$

- Bùi Quốc Tính ${ }^{2}$

- Trương Tích Thiện ${ }^{1}$

${ }^{1}$ Trường Đại học Bách Khoa, ĐHQG-HCM

2 Khoa Cơ và Tin học môi trường, Viện Công nghệ Tokyo, 2-12-1-W8-22, Ookayama, Meguro-ku, Tokyo, 152-8552, Nhật Bản

TÓM TÁT:

Phần tử 4 nút nội suy liên tiếp (CQ4) được mở rộng để giải bài toán truyền nhiệt hai chiều. Trong đó, giá trị gradient trung bình tại nút được sử dụng trong phương trình nội suy, dẫn tới hệ quả là lời giải có bậc cao hơn, dù không sử dụng thuật toán làm trơn và không làm

tăng số bậc tự do của hệ. Phương pháp đề nghị có thể dễ dàng được hiện thực hóa và tích hợp vào bất cứ mã nguồn FEM nào hiện có. Nhiều ví dụ số được xem xét để làm rõ tính chính xác và hiệu suất của phương pháp đề nghị trong việc phân tích truyền nhiệt hai chiều.

Từ khóa: truyền nhiệt, CFEM, dẫn nhiệt, đối lưu, gradient tại nút.

\section{REFERENCES}

[1]. Li E, Zhang Z, He Z. C., Xu X, Liu G. R. and $\mathrm{Li}$ Q., Smooth finite element method with exact solutions in heat transfer problems, International Journal of Heat and Mass Transfer 78 (2014) 1219-1231.

[2]. [2] Singh A., Singh I. V, Prakash R., Meshless element free Galerkin method for unsteady heat transfer problems, International Journal of Heat and Mass transfer 50 (2007) 1212-1219.

[3]. [3] Liu L. H. and Tan J. Y., Meshless local Petrov-Galerkin approach for coupled radiative and conductive heat transfer, International Journal of Thermal Sciences 46 (2007) 672-681.

[4]. [4] Khosravifard A., Hematiyan R. and Marin L., Nonlinear transient heat conduction analysis of functionally graded materials in the presence of heat sources using an improved radial point interpolation method, Applied Mathematical Modelling 35 (2011) 4157-4174.

[5]. [5] Cui X. Y, Feng S. Z. and Liu G. Y. - A cell-based smoothed radial interpolation method (CS-RPIM) for heat transfer analysis. Engineering Analysis with Boundary Elements 40 (2014) 147-153.

[6]. [6] Zheng, C., Wu S. C., Tang X. H. Tang and Zhang J. H., A novel twice-interpolation finite element method for solid mechanics problems, Acta Mechanica Sinica 26 (2010), 265-278.

[7]. [7] Bui Q. T, Vo Q. Đ., Zhang C. and Nguyen Đ. D., A consecutive-interpolation quadrilateral element (CQ4): Formulation and Application, Finite Element in Analysis and Design 84 (2014) 13-3. 